\title{
Interaction of dislocations with twinning boundary in bi-twinned metallic nanowires
}

Guangming Cheng ${ }^{1}$ and Yong $\mathrm{Zhu}^{2}$

${ }^{1}$ Princeton Institute for the Science and Technology of Materials, Princeton University, Princeton, New Jersey, United States, ${ }^{2}$ Department of Mechanical and Aerospace Engineering, North Carolina State University, Raleigh, North Carolina, United States

\section{Email: gcheng2@princeton.edu and yzhu@ncsu.edu}

Metallic NWs usually exhibit ultrahigh mechanical strength and are ideal candidates for studying fundamental deformation mechanisms at the nanoscale.[1-5] Metallic NWs with internal TBs have received much attention recently, such as metallic NWs with parallel, inclined or perpendicular TBs with respect to the NW length direction. Interaction between dislocations and TBs has become one of the most fundamental problems in materials science due to the emergence of nanotwinned materials over the past 2 decades. The twinned metallic NWs can provide an interesting platform to investigate the energetics and kinetics of dislocation-twin boundary interactions. Here, we report real-time observation of dislocation nucleation, interaction with the TBs and retraction, while simultaneously measuring the stress and strain in bi-twinned Ag NWs (with a single TB running parallel to the NW length direction) via quantitative in-situ transmission electron microscopy (TEM) tensile tests, using a state-of-the-art microelectromechanical system (MEMS) stage.[6-8] Our results show that leading partial dislocations nucleated from the free surface can be hindered by the TB, and upon unloading all or part of the leading partials can retract due to the repulsive force from the TB, leading to full or partial plastic strain recovery (Bauschinger effect), respectively.

Figure 1 shows the stress-strain responses and snapshots of microstructure evolution during typical loading-unloading cycles of a bi-twinned $\mathrm{Ag} \mathrm{NW}$ with or without a holding step. Note that the viewing direction in Fig. 1c,d is from the [1-10] zone axis as marked by the orange arrow in the inset in Fig. 1a. As a partial dislocation (marked by A in Fig. 4 c-ii) came out during loading (with the applied stress, 1.46 $\mathrm{GPa}$, slightly over the yield strength, $1.41 \mathrm{GPa}$ ), we immediately started unloading the NW by decreasing the applied force. It was observed that another partial (marked by B in Fig. 1c-iii) appeared at the initial stage of unloading. As the applied stress continued to decline, the later generated partial B first disappeared (Fig. 1c-iv) when the applied stress was decreased by 75\%. Subsequently, partial A also disappeared as the applied loading was fully released. At this point, the plastic strain was almost recovered after fully releasing the applied force. This suggests that, in the absence of an applied stress, the TB provides a repulsive force for the leading partials to retract and disappear. Fig. 1b,d displays a loading-unloading cycle with a short-time $(5 \mathrm{~min})$ stress relaxation under a fixed applied load. The results suggest that the internal TB in the bi-twinned NWs can act as an obstacle for hindering the propagation of partial dislocations across the TB in the time when the applied stress is slightly over the yield strength and facilitate retraction of the partials upon unloading. In comparison to the cases in bi-twinned NWs, the single crystalline NWs showed only elastic unloading (i.e., linear strain-stress behavior) and the planar sliding formed during loading was a permanent plastic deformation, as shown in Fig. 2. Therefore, there is no recoverable plasticity in single crystalline NWs.[9] 

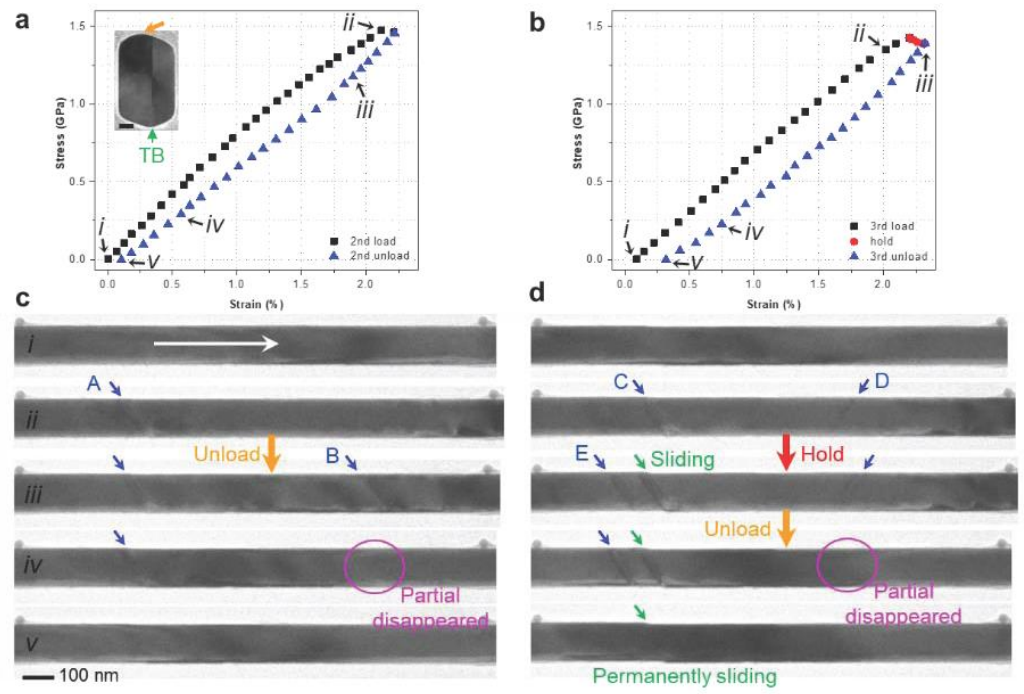

Figure 1. (a,b) Stress-strain curves for the bi-twinned Ag NW without or with a holding step, respectively. The relaxation step took $5 \mathrm{~min}$. Inset in (a) is the corresponding cross-sectional images of the tested NW. Scale bar, 20 $\mathrm{nm}$. (c,d) Snapshots of microstructure evolution during loading-unloading and loading-holding-unloading processes, respectively. The location of partial dislocations and planar slip in the NW are marked by blue and green arrows, respectively. Scale bar, $100 \mathrm{~nm}$.
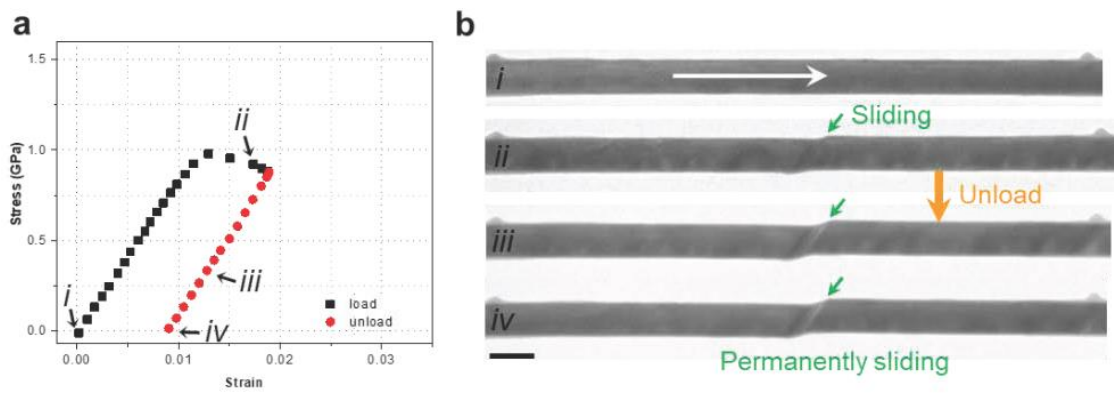

Figure 2. (a) Stress-strain curve for a single crystalline Ag NW under in-situ TEM tensile testing. (b) Snapshots of microstructure evolution during the loading-unloading process. Permanent planar sliding in the NW is marked by the green arrows. Scale bar, $100 \mathrm{~nm}$.

\section{References}

[1] S. Yin, G.M. Cheng, T.H. Chang, G. Richter, Y. Zhu, H.J. Gao, Hydrogen embrittlement in metallic nanowires, Nature Communications 10 (2019).

[2] S. Yin, G. Cheng, G. Richter, H. Gao, Y. Zhu, Transition of Deformation Mechanisms in SingleCrystalline Metallic Nanowires, Acs Nano 13(8) (2019) 9082-9090.

[3] Q. Qin, S. Yin, G. Cheng, X. Li, T.-H. Chang, G. Richter, Y. Zhu, H. Gao, Recoverable plasticity in penta-twinned metallic nanowires governed by dislocation nucleation and retraction, Nature Communications 6 (2015).

[4] G. Cheng, S. Yin, T.-H. Chang, G. Richter, H. Gao, Y. Zhu, Anomalous Tensile Detwinning in Twinned Nanowires, Physical Review Letters 19(25) (2017).

[5] S. Yin, G. Cheng, Y. Zhu, H. Gao, Competition between shear localization and tensile detwinning in twinned nanowires, PHYSICAL REVIEW MATERIALS 4 (2020) 023603. 
[6] G. Cheng, S. Yin, C. Li, T.-H. Chang, G. Richter, H. Gao, Y. Zhu, In-situ TEM study of dislocation interaction with twin boundary and retraction in twinned metallic nanowires, Acta Materialia 196 (2020) 304-312.

[7] G. Cheng, Y. Zhang, T.-H. Chang, Q. Liu, L. Chen, W.D. Lu, T. Zhu, Y. Zhu, In Situ Nanothermomechanical Experiment Reveals Brittle to Ductile Transition in Silicon Nanowires, Nano Letters 19(8) (2019) 5327-5334.

[8] G. Cheng, S. Yao, X. Sang, B. Hao, D. Zhang, Y.K. Yap, Y. Zhu, Evolution of Irradiation-Induced Vacancy Defects in Boron Nitride Nanotubes, Small 12(6) (2016) 818-824.

[9] The authors acknowledge funding from the National Science Foundation (NSF) under Award No. CMMI-1762511, and the use of the Analytical Instrumentation Facility (AIF) at North Carolina State University (NSF Award No. ECCS-1542015). 